\title{
Positive Medication Changes Resulting from Comprehensive and Noncomprehensive Medication Reviews in a Medicare Part D Population
}

\author{
Allison Buhl, PharmD; Jill Augustine, PharmD, MPH, PhD; Ann M. Taylor, MPH, MCHES;
} Rose Martin, PharmD; and Terri L. Warholak, PhD, RPh, CPQA

\begin{abstract}
BACKGROUND: Health care organizations face the challenge of reducing costs while improving health outcomes. Currently, more than 39 million seniors are enrolled in a Medicare Part D prescription benefit plan, many of whom also qualify for medication therapy management (MTM) services. MTM programs provide valuable services designed to prevent or resolve medication-related problems (MRPs). Two core components of all MTM programs include comprehensive medication reviews (CMRs) with followup interventions and focused non-CMR interventions. Currently, there is limited research comparing the rate of MRPs resolved by CMR and nonCMR interventions.
\end{abstract}

OBJECTIVE: To determine whether CMRs versus non-CMR interventions resulted in more pharmacist-initiated intervention recommendations and positive medication changes.

METHODS: This retrospective, cross-sectional quality improvement project evaluated outcomes for individuals who received interventions following a CMR compared with those who received non-CMR interventions as part of a comprehensive MTM program. For this project, eligible individuals qualified for an MTM program. A positive medication change was defined as the addition of an appropriate medication or the removal of an inappropriate medication within 120 days of a pharmacist recommendation. Differences between the groups were calculated using the Wilcoxon rank sum test.

RESULTS: During the 2-year period (2012 and 2013), 788,756 beneficiaries were enrolled in the MTM program and evaluated for potential MRPs. In both years, pharmacists recommended more medication changes per member for those receiving a CMR (0.81 in 2012 and 0.82 in 2013) compared with the non-CMR group (0.68 in 2012 and 0.61 in 2013; $P$ value $<0.001$ for both years). However, recommendations made via non-CMR interventions (representing the vast majority of all medication reviews) were more likely to result in a positive medication change $(0 \mathrm{R}=1.24,95 \% \mathrm{Cl}=1.21-1.28$ for 2012; $\mathrm{OR}=1.26,95 \% \mathrm{Cl}=1.22-1.30$ for 2013).

CONCLUSIONS: These quality improvement project results suggest that Medicare Part D beneficiaries participating in a university-based MTM program who received non-CMR interventions had a higher likelihood of having positive medication changes than those receiving CMRs. These results are enlightening and may provide initial evidence to support inclusion of a nonCMR performance metric for the Centers for Medicare \& Medicaid Service's star ratings for MTM programs.

J Manag Care Spec Pharm. 2017;23(3):388-94

Copyright $\odot 2017$, Academy of Managed Care Pharmacy. All rights reserved.

\section{What is already known about this subject}

The Centers for Medicare \& Medicaid Services (CMS) requires medication therapy management (MTM) programs to provide targeted medication reviews and comprehensive medication reviews (CMRs) to eligible beneficiaries meeting minimum criteria.

Targeted medication reviews assess beneficiaries' medication use to help identify medication-related problems and are subsequently addressed either by CMRs or non-CMR interventions. CMS includes the annual percentage of beneficiaries receiving a CMR in its star ratings for MTM programs yet lacks a metric for tracking those receiving a non-CMR intervention.

\section{What this study adds}

This large-scale quality improvement project compared CMR and non-CMR interventions to determine which was more effective in producing positive medication changes in a Medicare Part D population enrolled in MTM.

Findings from this project indicate that non-CMR interventions were more effective overall and may provide initial evidence to support inclusion of a non-CMR performance metric for CMS star ratings for MTM programs.

T he elderly population is expanding rapidly, placing greater demands on the health care system than ever before. Seniors are the largest consumers of health care resources and fill close to one third of all prescriptions. ${ }^{1}$ Furthermore, most seniors have at least 1 chronic medical condition, and over half take 5 or more medications regularly.,2 Generally, as the number of medications taken increases, patient adherence to the prescribed regimen decreases. ${ }^{3}$ Data suggest that only about $50 \%$ of patients with chronic conditions take their medications consistently as directed. ${ }^{4}$

In 2006, the Centers for Medicare \& Medicaid Services (CMS) launched the current Medicare Part D prescription benefit program to help beneficiaries afford necessary medications for managing their chronic conditions. As a program requirement, Medicare Part D sponsors must provide medication therapy management (MTM) services to eligible beneficiaries in an effort to reduce adverse events and ensure optimum 
therapeutic outcomes. ${ }^{5}$ MTM eligibility standards are established by CMS on an annual basis, and Medicare Part D sponsors then select their parameters based on these criteria. This project included Medicare Part D sample populations from the 2012 and 2013 calendar years. The 2012 and 2013 MTM qualification standards included beneficiaries who (a) had at least 2 chronic conditions; (b) were taking at least 3 chronic medications; and (c) met the minimum mandatory annual drug spend (\$3,100 and \$3,144 in 2012 and 2013, respectively). ${ }^{6}$

MTM programs are required to perform targeted medication reviews at least quarterly and offer annual comprehensive medication reviews (CMRs) to all MTM-qualified beneficiaries. A targeted medication review is a systematic review of an individual's medication profile, designed to identify the following: (a) potential medication-related problems (MRPs; e.g., safety concerns such as inappropriate use of certain medications in the elderly, drug-drug interactions, and therapeutic duplications); (b) use of higher-cost agents when alternative medications are available (e.g., lower-cost generics); and (c) absence of guidelines-based therapeutic agents (e.g., statins in qualifying diabetic patients).

For the university-based MTM program evaluated for this study, the MRPs identified during a targeted medication review were subsequently addressed via a CMR or non-CMR interventions. CMRs are person-to-person consultations that include a review of the beneficiary's allergies and medical history, a complete medication reconciliation, and an assessment and review of therapeutic goals. The qualified MTM provider performing the CMR (a pharmacist or pharmacy intern acting under the supervision of a pharmacist) creates a medication action plan and personal medication list that is given to the beneficiary following the CMR. Additionally, the pharmacist contacts the beneficiary's prescriber with recommendations to modify treatment to address any identified medication-related problems. In contrast, non-CMRs involve shorter and more focused discussions, written beneficiary outreach, and/or direct provider outreach to address problems. Non-CMR interventions are performed for beneficiaries unable or unwilling to complete a CMR or in cases where the intervention is time sensitive and prescriber outreach must occur quickly.

Research shows that MTM services are effective in decreasing adverse medication events and hospital readmission rates and that pharmacist-delivered MTM medication education decreases health care use and overall expenditures. ${ }^{7-16}$ However, the published literature comparing CMRs and non-CMR interventions is limited. To date, most studies have focused solely on CMRs. Perlroth et al. (2013) found that annual CMRs were effective in producing positive outcomes (e.g., improved prescribing quality in MTM populations). ${ }^{17}$ In addition, Isetts et al. (2008) reported that face-to-face MTM services were effective in resolving medication-related problems without prescriber involvement. ${ }^{16}$ Roane et al. (2014) found that an electronic record review in tandem with a telephonic MTM program resulted in a more thorough assessment of patient therapy. ${ }^{18}$ Finally, Chinthammit et al. (2015) showed that non-CMR interventions were less costly and more effective than CMRs. ${ }^{19}$

CMRs can be highly beneficial, yet they typically incorporate an extensive amount of information that may overwhelm some beneficiaries. Educational research suggests that simplified and repeated messaging may facilitate more effective learning of complex information. ${ }^{20}$ Non-CMR interventions are designed to address specific prioritized problems, so beneficiaries may find this type of intervention less intimidating, since it allows for more concise and focused education. However, the only CMS star metric for MTM programs is the percentage of qualified beneficiaries receiving a CMR in a given calendar year. ${ }^{3}$ Presently, no performance measure exists for non-CMR interventions.

Currently, more than 39 million seniors are enrolled in the Medicare Part D program, ${ }^{21}$ and approximately $11 \%$ of those seniors are enrolled in an MTM program. ${ }^{22}$ In 2015, CMS released the first star cut points for the CMR completion rate measure, thereby placing additional emphasis on this metric While CMS uses star measures pertaining to use of guidelinesbased therapy and adherence, it is important to note that there are no current measures for resolution of MRPs for CMRs or non-CMR interventions. As health care organizations strive to reduce medication costs while simultaneously improving patient health outcomes, it is apparent that more research is needed to determine the optimal approach for accomplishing this task.

The purpose of this quality improvement project was to compare CMR versus non-CMR interventions for patients receiving services via a university-based MTM program to determine which approach achieved more pharmacist-initiated intervention recommendations and more positive medication changes.

\section{Methods}

\section{Project Design}

This retrospective, cross-sectional, observational project compared the number of interventions and positive medication changes resulting from $\mathrm{CMR}$ versus non-CMR interventions in a Medicare Part D population receiving MTM services from a university-based program. The university's institutional review board deemed this a quality improvement project.

\section{Target Population}

The target population was beneficiaries who received MTM services from the University of Arizona Medication Management Center (UAMMC), which serves beneficiaries nationwide who qualify for MTM services through Medicare Part D. Inclusion criteria for this project were beneficiaries who qualified for MTM services based on their Medicare Part D sponsor's criteria and filled medications during the 2012 or 2013 calendar year. A positive medication change was defined 
TABLE 1 Sociodemographic and Health-Related Characteristics of Medicare Part D Beneficiaries Who Received a CMR or Non-CMR Intervention

\begin{tabular}{|c|c|c|c|c|c|c|}
\hline \multirow[b]{3}{*}{ Characteristic } & \multicolumn{3}{|c|}{2012} & \multicolumn{3}{|c|}{2013} \\
\hline & \multirow{2}{*}{$\begin{array}{c}\text { CMR Group } \\
(\mathrm{n}=42,253) \\
\mathrm{n}(\%)\end{array}$} & \multirow{2}{*}{$\begin{array}{c}\begin{array}{c}\text { Non-CMR } \\
(\mathrm{n}=384,477)\end{array} \\
\mathrm{n}(\%) \\
\end{array}$} & \multirow[b]{2}{*}{$P$ Value ${ }^{\mathrm{a}}$} & \multirow{2}{*}{$\begin{array}{c}\begin{array}{c}\text { CMR Group } \\
(\mathrm{n}=38,967)\end{array} \\
\mathrm{n}(\%)\end{array}$} & \multirow{2}{*}{$\begin{array}{c}\begin{array}{c}\text { Non-CMR } \\
(\mathrm{n}=342,701)\end{array} \\
\mathrm{n}(\%) \\
\end{array}$} & \multirow[b]{2}{*}{$P$ Value $^{\mathrm{a}}$} \\
\hline & & & & & & \\
\hline \multicolumn{7}{|l|}{ Gender } \\
\hline Female & $26,740 \quad(63)$ & 232,222 & \multirow{2}{*}{$<0.001$} & 22,895 & 201,750 & \multirow{2}{*}{0.65} \\
\hline Male & $15,513 \quad(37)$ & $152,255 \quad(40)$ & & 16,072 & 140,936 & \\
\hline \multicolumn{7}{|c|}{ Chronic disease $^{b}$} \\
\hline Hypertension & 38,405 & 357,466 & & 35,321 & 316,367 & \\
\hline Dyslipidemia & 30,854 & 289,591 & & 28,605 & 247,457 & \\
\hline Diabetes & 24,426 & 204,681 & & 20,396 & 153,895 & \\
\hline Depression & 13,676 & 135,709 & & 10,560 & 105,131 & \\
\hline Heart failure & 9,237 & 93,929 & & 8,318 & 78,692 & \\
\hline Respiratory & $10,880 \quad(26)$ & 90,017 & & 8,941 & 79,135 & \\
\hline Osteoporosis & 6,025 & 53,362 & & 3,950 & 37,075 & \\
\hline \multicolumn{7}{|c|}{ Dollars spent on prescription medications (U.S. dollars) } \\
\hline Mean (SD) & $1,912(2,511)$ & $1,871(2,423)$ & \multirow{2}{*}{0.001} & $2,192(4,011)$ & \multirow{2}{*}{$\begin{array}{l}2,071(3,250) \\
1,350(1,214)\end{array}$} & \multirow{2}{*}{$<0.001$} \\
\hline Median (IQR) & $1,315(1,101)$ & $1,291(1,075)$ & & $1,391(1,277)$ & & \\
\hline \multicolumn{7}{|c|}{ Number of chronic medications } \\
\hline Mean (SD) & $10.7 \quad(3.5)$ & $10.8 \quad(3.6)$ & \multirow{2}{*}{$<0.001$} & $(4.7)$ & $11.2 \quad(4.8)$ & \multirow{2}{*}{0.05} \\
\hline Median (IQR) & $(4)$ & $(5)$ & & $(5)$ & $(5)$ & \\
\hline
\end{tabular}

as the addition of an appropriate medication or the removal of an inappropriate medication within 120 days of a pharmacist recommendation, as determined by electronic review of claims data. Retrospective group assignment was based on the type of intervention received (CMR or non-CMR).

\section{Data Collection}

Each beneficiary who qualified for MTM services received a targeted medication review to assess for potential MRPs every time a prescription claim was processed and every time a prescription claim refill was expected for a chronic medication. Targeted medication reviews were conducted via electronic evaluation of claims data. When a medicationrelated problem was identified, a UAMMC staff member (i.e., pharmacist, pharmacy intern, or pharmacy technician) contacted the patient by telephone to initiate a CMR. If the beneficiary was unreachable, unwilling, or unable to complete a CMR, a staff member completed a non-CMR intervention such as a brief phone discussion focused solely on the potential MRPs, a letter sent to the beneficiary, and/or a phone call or facsimile sent to the prescriber.

The intervention method was selected systematically based on the type and severity of the problem, according to a preset algorithmic ranking system. Implementation of a non-CMR intervention could also occur if the problem identified was time sensitive or severe (e.g., a potentially serious drugdrug interaction between warfarin and azithromycin) and required immediate outreach to the beneficiary's prescriber. The UAMMC staff documented the type of review performed (CMR or non-CMR), the number of each type of intervention recommended to providers, and the number of intervention recommendations accepted by providers (i.e., positive medication changes). Beneficiaries who had multiple intervention recommendations sent to their providers or multiple positive medication changes were only included in the dataset once. Intervention recommendation data were collected to demonstrate the number of MRPs identified through the present algorithms and the number addressed by pharmacists.

The data collected also show the effect of pharmacistprovided medication reviews on ensuring that patients receive the correct medication therapy and reduce medication-related problems. In addition, the UAMMC software system collected beneficiary information including demographics, medical claims data, prescriptions processed through Medicare Part D, and self-reported information (e.g., medications, allergies, and conditions). For inclusion in the final datasets, beneficiaries also had to meet CMS 2012 and 2013 eligibility criteria to receive MTM services (e.g., having at least 2 chronic conditions; taking at least 3 chronic medications; and meeting mandated annual drug cost (\$3,100 in 2012 and $\$ 3,144$ in 2013). 
TABLE 2 Beneficiaries Who Received an Intervention Recommendation and Positive Medication Change by Group and Project Year

\begin{tabular}{|c|c|c|c|c|c|c|}
\hline & \multicolumn{3}{|c|}{2012} & \multicolumn{2}{|c|}{2013} & \multirow[b]{2}{*}{$P$ Value } \\
\hline & $\begin{array}{l}\text { CMR Group } \\
(n=43,490)\end{array}$ & $\begin{array}{l}\text { Non-CMR Group } \\
\quad(\mathbf{n}=375,159)\end{array}$ & $P$ Value & $\begin{array}{l}\text { CMR Group } \\
(\mathrm{n}=38,101)\end{array}$ & $\begin{array}{l}\text { Non-CMR Group } \\
(\mathbf{n}=332,006)\end{array}$ & \\
\hline \multicolumn{7}{|c|}{ Number of intervention recommendations, $\mathbf{n}(\%)^{\mathrm{a}}$} \\
\hline None & $19,259 \quad(44)$ & $199,143 \quad(53)$ & \multirow{5}{*}{$<0.001$} & $18,012 \quad(47)$ & $196,873 \quad(59)$ & \multirow{5}{*}{$<0.001$} \\
\hline 1 & $16,141 \quad(37)$ & $118,620 \quad(32)$ & & $12,505 \quad(33)$ & $87,973 \quad(25)$ & \\
\hline 2 & $5,876 \quad(14)$ & $42,050 \quad(11)$ & & $5,092 \quad(13)$ & $33,037 \quad(10)$ & \\
\hline 3 & $1,691 \quad(4)$ & $11,682 \quad(3)$ & & $1,764 \quad(5)$ & $10,235 \quad(3)$ & \\
\hline 4 or more & $523 \quad(1)$ & $3,664 \quad(1)$ & & $728 \quad(2)$ & $3,888 \quad(1)$ & \\
\hline \multicolumn{7}{|c|}{ Number of positive medication changes, ${ }^{b} \mathbf{n}(\%)^{a}$} \\
\hline No changes & $35,778 \quad(82)$ & $310,464 \quad(83)$ & \multirow{5}{*}{$<0.001$} & $31,067 \quad(82)$ & $277,035 \quad(83)$ & \multirow{5}{*}{$<0.001$} \\
\hline 1 & $6,108 \quad(14)$ & $47,417 \quad(13)$ & & $5,747 \quad(15)$ & $43,932 \quad(13)$ & \\
\hline 2 & $1,243 \quad(3)$ & $12,617 \quad$ (3) & & $1,085 \quad(3)$ & $9,011 \quad(3)$ & \\
\hline 3 & $278 \quad(1)$ & $3,379 \quad(1)$ & & $172 \quad(1)$ & $1,657 \quad(1)$ & \\
\hline 4 or more & $83(0.2)$ & $1,282 \quad(0.3)$ & & $30(0.1)$ & $371 \quad(0.1)$ & \\
\hline \multicolumn{7}{|c|}{$\begin{array}{l}\text { aPercentages may not equal } 100 \% \text { due to rounding. } \\
\text { bA positive medication change was defined as the addition of an appropriate medication or the removal of an inappropriate medication within } 120 \text { days of a pharmacis } \\
\text { recommendation. }\end{array}$} \\
\hline
\end{tabular}

Demographic information was not part of the initial data collection, since this was a retrospective project. Thus, the baseline demographic characteristics were collected at a different time and include all beneficiaries that met the CMS eligibility criteria for that year.

\section{Data Analysis}

Data analysis was conducted using STATA SE, version 12.1 (StataCorp, College Station, TX). The number of recommendations and medication changes per member were calculated by dividing the sum total of prescriber interventions (or medication changes) by the number of beneficiaries for each group (CMR or non-CMR) for each project year (2012 or 2013). Demographic characteristics were compared using chi-square tests. Wilcoxon rank sum tests were used to determine differences in the total recommendations and total positive medication changes attained between beneficiaries receiving CMRs versus those receiving non-CMR interventions for each calendar year. Odds ratios were calculated to determine the odds of receiving a prescriber intervention or medication change for each calendar year. A two-tailed a priori alpha level of 0.05 was used.

\section{Results}

A total of 808,398 beneficiaries met the project's inclusion criteria (426,730 in 2012 and 381,668 in 2013). A total of 19,642 beneficiaries were excluded because they declined MTM services with their insurance company or were disenrolled by their insurance provider. Thus, the remaining 788,756 eligible beneficiaries were included in the CMR versus non-CMR calculations during the 2-year project period: 418,649 beneficiaries in 2012 and 370,107 in 2013.
In the 2012 calendar year, the majority (61\%) of beneficiaries were female. Beneficiaries filled 10 chronic medication prescriptions on average, totaling $\$ 1,874.00$ (standard deviation [SD] \$2,431.59) per quarter. The most frequently reported chronic conditions were hypertension (93\%), dyslipidemia (75\%), and diabetes (54\%; Table 1). Out-of-pocket medication costs were higher for CMR recipients (mean $\$ 1,911.88$ [SD \$2,510.67]) compared with non-CMR recipients (mean $\$ 1,870.90$ [SD $\$ 2,422.71$ ]; $P=0.001)$. However, CMR beneficiaries filled fewer medications (mean 10.7 [SD 3.49]) than nonCMR beneficiaries (mean 10.8 [SD 3.58]; $P<0.001$ ).

In the 2013 calendar year, there were more (59\%) female beneficiaries than male. On average, beneficiaries filled 11 chronic medication prescriptions, totaling \$2,083.05 (SD \$3,336.21) per quarter. The top comorbid conditions were hypertension (92\%), dyslipidemia (72\%), and diabetes (46\%). CMR recipients had a higher out-of-pocket medication cost (mean \$2,191.75 [SD \$4,011.42]) compared with non-CMR recipients (mean $\$ 2,070.69$ [SD \$3,250.34]; $P<0.001)$. However, there were no differences in number of medications taken between groups.

In 2012 and 2013, the majority of beneficiaries in both groups received either zero or 1 recommendation; less than $2 \%$ in either group received 4 or more recommendations. Overall, nearly $20 \%$ of beneficiaries had a positive medication change as a result of receiving either type of intervention (Table 2).

In 2012, those beneficiaries in the CMR group received significantly more intervention recommendations per member than those in the non-CMR group $(0.809$ vs. $0.677 ; P<0.001)$. There were no significant differences in the number of medication changes per member $(0.236$ for the CMR group vs. 0.225 for the non-CMR group; $P=0.16$ ) between the groups. 
TABLE 3 Total Number of Intervention Recommendations and Medication Changes per Member by Group and Project Year

\begin{tabular}{|c|c|c|c|c|c|c|c|c|}
\hline & \multicolumn{4}{|c|}{2012} & \multicolumn{4}{|c|}{2013} \\
\hline & $\begin{array}{l}\text { CMR } \\
\text { Group }\end{array}$ & $\begin{array}{c}\text { Non-CMR } \\
\text { Group }\end{array}$ & Total & \multirow[b]{2}{*}{$P$ Value $^{\mathrm{a}}$} & $\begin{array}{c}\text { CMR } \\
\text { Group }\end{array}$ & $\begin{array}{c}\text { Non-CMR } \\
\text { Group }\end{array}$ & Total & \multirow[b]{2}{*}{$P$ Value $^{\mathrm{a}}$} \\
\hline & $\mathrm{n}$ & $\mathbf{n}$ & $\mathrm{n}$ & & $\mathbf{n}$ & $\mathbf{n}$ & $\mathrm{n}$ & \\
\hline Members & 43,490 & 375,159 & 418,649 & & 38,101 & 332,006 & 370,107 & \\
\hline Intervention recommendations $^{\mathrm{b}}$ & 35,207 & 253,494 & 288,701 & & 31,181 & 201,670 & 232,851 & \\
\hline Intervention recommendations per memberc & 0.809 & 0.678 & 0.687 & $<0.001$ & 0.818 & 0.608 & 0.632 & $<0.001$ \\
\hline Medication changes $^{\mathrm{b}}$ & 9,796 & 88,467 & 98,263 & & 8,559 & 68,477 & 77,036 & \\
\hline Medication changes per memberc & 0.225 & 0.236 & 0.230 & 0.160 & 0.225 & 0.207 & 0.210 & $<0.001$ \\
\hline \multicolumn{9}{|c|}{$\begin{array}{l}\text { aP values indicate the comparison between CMR group and non-CMR group for number of interventions per member and the number of medication changes per member } \\
\text { by project year. } \\
\text { bPrescriber interventions and medication changes reflect total sum. }\end{array}$} \\
\hline
\end{tabular}

In 2013, similar to 2012, the CMR group received significantly more recommendations per member than those in the non-CMR intervention group ( 0.818 vs. $0.608 ; P<0.001)$. Additionally, there was a significant difference in the number of medication changes per member between the CMR and nonCMR groups ( 0.225 vs. $0.207 ; P<0.001$; Table 3 ).

CMR recipients were more likely to receive an intervention recommendation than non-CMR recipients (2012: OR $=0.70$, 95\% CI $=0.69-0.72 ; \quad 2013: \quad O R=0.62,95 \% \quad C I=0.60-0.63)$. However, in both years, the odds of having a positive medication change was higher in the non-CMR group compared with the CMR group (2012: OR $=1.24,95 \% \mathrm{CI}=1.21-1.28 ; 2013$ : $\mathrm{OR}=1.26,95 \% \mathrm{CI}=1.22-1.30$; Table 4).

\section{Discussion}

In the 2012 and 2013 samples, the CMR group was significantly more likely than the non-CMR group to have an intervention recommendation sent to their providers. While CMRs allow pharmacists to probe in depth regarding beneficiaries' medication use, non-CMR interventions are more focused and typically only address any MRPs identified by the targeted medication review using nonpatient-reported information. Consequently, CMRs may result in identification of additional MRPs that may not be noted during non-CMRs. However, both interventions are clearly effective in resolving MRPs that lead to unnecessary health expenditures.

This quality improvement project found that beneficiaries in the non-CMR group received fewer intervention recommendations per person; yet, the recommendations made were more likely to result in positive medication changes. This parallels the literature, which shows that a focused approach to medication changes may be more effective. ${ }^{24}$ In the 2012 nonCMR sample, the vast majority of recommendations accepted by prescribers involved guideline-based recommendations (e.g., addition of an angiotensin-converting enzyme inhibitor for a hypertensive diabetic) or safety recommendations (e.g., discontinuation of duplicative therapy). ${ }^{23}$ Conversely, CMRs generated other interventions (e.g., potential adverse reactions to medications) based on the pharmacist's individual analysis of the beneficiary's medication use. This intrinsic difference in recommendation type may account for the higher incidence of provider acceptance of recommendations made via non-CMR interventions.

A statistically significant difference in the number of medication changes per member was observed between the 2 groups in 2013, perhaps because of the large sample; however, it may not represent an appreciable difference between the types of interventions. While non-CMR recipients comprised the majority of the sample, they also received the majority of medication changes.

In today's MTM landscape, providers focus much more heavily on completing CMRs because they are the only type of intervention recognized by CMS in its star ratings metric for MTM programs. However, recent research suggests that non-CMR interventions are more cost effective than CMRs. ${ }^{24}$ The results presented here parallel those of another study, ${ }^{25}$ and they provide initial evidence that non-CMR interventions offer a viable avenue to address medication-related problems, reduce adverse drugs events, and minimize medication costs for patients and insurance payers (notably CMS). While still preliminary, these results represent important information for MTM pharmacists, providers, and regulatory officials as they strive strive to improve patient safety and outcomes while reducing health care costs.

\section{Limitations}

It is important to acknowledge several limitations of this quality improvement project. First, the UAMMC aims to maximize CMR rates in accordance with the CMS performance metric. Beneficiaries who receive CMRs may inherently differ from 
Positive Medication Changes Resulting from Comprehensive and Noncomprehensive Medication Reviews in a Medicare Part D Population

TABLE 4 Odds Ratio Analysis Comparing Odds of Receiving a Prescriber Intervention or Medication Change for CMR Recipients by Project Year

\begin{tabular}{|c|c|c|c|c|c|c|}
\hline & \multicolumn{3}{|c|}{2012} & \multicolumn{3}{|c|}{2013} \\
\hline & CMR Group & Non-CMR Group & OR $(95 \% \mathrm{CI})$ & CMR Group & Non-CMR Group & OR $(95 \% \mathrm{CI})$ \\
\hline & $\mathrm{n}$ & $\mathbf{n}$ & & $\mathbf{n}$ & $\mathbf{n}$ & \\
\hline Intervention recommendation & 24,231 & 176,016 & $0.70(0.69-0.72)$ & 20,089 & 135,133 & $0.62(0.60-0.63)$ \\
\hline No intervention recommendation & 192,259 & 199,259 & & 18,012 & 196,873 & \\
\hline Medication change $^{\mathrm{a}}$ & 7,369 & 61,969 & $1.24(1.21-1.28)$ & 7,034 & 54,971 & $1.26(1.22-1.30)$ \\
\hline No medication change & 16,862 & 114,047 & & 13,055 & 80,182 & \\
\hline
\end{tabular}

those receiving non-CMR interventions, and beneficiaries receiving non-CMRs may be unable or unwilling to complete the in-depth review.

Second, in the 2012 and 2013 samples, there were significant differences in the sociodemographic characteristics of the groups at baseline (gender, dollars spent on prescription medications, and number of concomitant medications). It is reasonable to assume that the difference in the female-male ratio of these Medicare Part D beneficiaries mirrors the national statistics for this more senior group. ${ }^{26}$ The reason for the observed differences in the other baseline characteristics is unclear. Further investigation is warranted to gain a better understanding of these differences.

Third, in the 2012 and 2013 samples, it is possible that the observed differences in average dollars spent and concomitant medications is related to individuals for whom MTM was designed (e.g., beneficiaries taking more than 3 chronic medications and incurring higher annual medication costs); the large sample sizes may also help account for some of these sociodemographic differences. Despite these observed differences, the results indicate that higher-risk beneficiaries are indeed receiving the additional support needed for their greater medication use and associated costs.

Fourth, the data used for this quality improvement project were initially included as part of a mandatory annual report required by CMS for all MTM programs. These reports included beneficiaries who qualified for MTM services and met additional requirements. However, some beneficiaries were excluded from these reports based on criteria established by CMS: excluded individuals were not enrolled or participating within the previous 60 days or opted out of receiving MTM services with their insurance company. Therefore, the demographic data used for this study included all beneficiaries who qualified for MTM services regardless of inclusion in the CMS annual reports. Consequently, the demographic sample was slightly larger (e.g., for 2012, 426,730 vs. 418,649 included in the CMS annual report) and may have differed slightly from the dataset analyzed when comparing the CMR and non-CMR groups.
Finally, the recommendations resulting from CMRs may differ inherently from those made for non-CMR recipients. The primary difference is potentially attributable to additional information obtained during CMRs compared with the information available through non-CMR interventions. These intrinsic differences may have had an effect on the number of medicationrelated problems addressed in each of the groups.

\section{Conclusions}

This quality improvement project found that non-CMR interventions resulted in fewer overall intervention recommendations but more positive medication changes for a Medicare Part D population participating in a university-based MTM program. While these findings are promising, further research is warranted to determine their replicability in more diverse populations and in other MTM settings (e.g., face to face or clinic based). These results provide initial evidence to support inclusion of non-CMR interventions as a performance metric for the CMS MTM star rating program.

\section{Authors}

ALLISON BUHL, PharmD, and ROSE MARTIN, PharmD, University of Arizona Medication Management Center, Tucson, Arizona. JILL AUGUSTINE, PharmD, MPH, PhD; ANN M. TAYLOR, MPH, MCHES; and TERRI L. WARHOLAK, PhD, RPh, CPQA, University of Arizona College of Pharmacy, Tucson, Arizona.

AUTHOR CORRESPONDENCE: Allison Buhl, PharmD, Medication Management Specialist, University of Arizona Medication Management Center, USA Bldg. 300B, Rm. B113, P.O. Box 210300, Tucson, AZ 85721. Tel.: 844.866.3730; E-mail: buhl@pharmacy.arizona.edu.

\section{DISCLOSURES}

No outside funding supported this study. The University of Arizona Medication Management Center (UAMMC)/SinfoniaRx provides grant funding to the University of Arizona for research. Augustine reports support from the UAMMC and ownership of Pfizer stock and was a graduate student at the University of Arizona College of Pharmacy at the time of this study. The authors report no other potential conflicts of interest. 
Buhl and Augustine contributed study concept and design, collected the data, and wrote the manuscript. Data interpretation was performed by all the authors. Taylor, Warholak, Augustine, and Martin revised the manuscript.

\section{REFERENCES}

1. Centers for Disease Control and Prevention, The Merck Institute for Aging and Health. The state of aging \& health in America 2004. Available at: https://www.cdc.gov/aging/pdf/State_of_Aging_and_Health_in_ America_2004.pdf. Accessed January 28, 2017.

2. Kaufman DW, Kelly JP, Rosenberg L, et al. Recent patterns of medication use in the ambulatory adult population of the United States: the Slone survey. JAMA. 2002;287(3):337-44.

3. Vlasnik JJ, Aliotta SL, DeLor B. Medication adherence: factors influencing compliance with prescribed medication plans. Case Manager. 2005;16(2):47-51.

4. Dimatteo RM, Giordani PJ, Lepper HS, Croghan TW. Patient adherence and medical treatment outcomes: a meta-analysis. Med Care. 2002;40(9): 794-811.

5. Centers for Medicare $\&$ Medicaid Services. Announcement of calendar year (CY) 2014 Medicare Advantage capitation rates and Medicare Advantage and Part D payment policies and final call letter. April 1, 2013. Available at: http://www.cms.gov/Medicare/Health-Plans/MedicareAdvtgSpecRateStats/ Downloads/Announcement2014.pdf. Accessed January 28, 2017.

6. Centers for Medicare \& Medicaid Services. 2012 and 2013 plan MTM program eligibility information. Available at: https://www.cms.gov/ Medicare/Prescription-Drug-Coverage/PrescriptionDrugCovContra/ Downloads/CY2012-MTM-Fact-Sheet.pdf and https://www.cms.gov/medicare/prescription-drug-coverage/prescriptiondrugcovcontra/downloads/ cy2013-mtm-fact-sheet.pdf. Accessed February 7, 2017.

7. Vira T, Colquhoun M, Etchells E. Reconcilable differences: correcting medication errors at hospital admission and discharge. Qual Saf Health Care. 2006;15(2):122-26

8. Nickerson A, MacKinnon NJ, Roberts N, Saulnier L. Drug-therapy problems, inconsistencies and omissions identified during a medication reconciliation and seamless care service. Healthc Q. 2005;8(Spec No):65-72.

9. Pronovost P, Weast B, Schwarz M, et al. Medication reconciliation: a practical tool to reduce the risk of medication errors. J Crit Care. 2003;18(4):201-05.

10. Rodehaver C, Fearing D. Medication reconciliation in acute care: ensuring an accurate drug regimen on admission and discharge. Jt Comm J Qual Beneficiary Saf. 2005;31(7):406-13.

11. Paquette-Lamontagne N, McLean W, Besse L, Cusson J. Evaluation of a new integrated discharge prescription form. Ann Pharmacother. 2001;35(7-8):953-58.

12. Ensing HT, Stuit CCM, van den Bemt BJ, et al. Identifying the optimal role for pharmacists in care transitions: a systematic review. J Manag Care Spec Pharm. 2015;21(8):614-38. Available at: http://www.jmcp.org/ doi/10.18553/jmcp.2015.21.8.614.

13. Iyer R, Coderre P, McKelvey T, et al. An employer-based, pharmacist intervention model for patients with type 2 diabetes. Am J Health Syst Pharm. 2010;67(4):312-15.
14. Barnett MJ, Frank J, Wehring H, et al. Analysis of pharmacist-provided medication therapy management (MTM) services in community pharmacies over 7 years. J Manag Care Pharm. 2009;15(1):18-31. Available at: http:// www.jmcp.org/doi/10.18553/jmcp.2009.15.1.18.

15. Wittayanukorn S, Westrick SC, Hansen RA, et al. Evaluation of medication therapy management services for patients with cardiovascular disease in a self-insured employer health plan. J Manag Care Pharm. 2013;19(5):385-95. Available at: http://www.jmcp.org/doi/abs/10.18553/jmcp.2013.19.5.385.

16. Isetts BJ, Schondelmeyer SW, Artz MB, et al. Clinical and economic outcomes of medication therapy management services: the Minnesota experience. J Am Pharm Assoc (2003). 2008;48(2):203-11.

17. Perlroth D, Marrufo G, Montesinos A, et al. Medication therapy management in chronically ill populations: final report. Center for Medicare \& Medicaid Innovation. August 2013. Available at: https://innovation.cms.gov/ files/reports/mtm_final_report.pdf. Accessed January 28, 2017.

18. Roane TE, Patel V, Hardin H, et al. Discrepancies identified with the use of prescription claims and diagnostic billing data following a comprehensive medication review. J Manag Care Pharm. 2014;20(2):165-73. Available at: http://www.jmcp.org/doi/10.18553/jmcp.2014.20.2.165.

19. Chinthammit C, Armstrong EP, Boesen K, Martin R, Taylor AM, Warholak T. Cost-effectiveness of comprehensive medication reviews versus noncomprehensive medication review interventions and subsequent successful medication changes in a Medicare Part D population. J Manag Care Spec Pharm. 2015;21(5):381-89. Available at: http://www.jmcp.org/doi/10.18553/ jmcp.2015.21.5.381.

20. Kolb D. Experiential Learning: Experience as the Source of Learning and Development. Upper Saddle River, NJ: Prentice Hall; 1984.

21. Hoadley J, Cubanski J, Neuman T. Medicare Part D at ten years: the 2015 marketplace and key trends, 2006-2015. October 5, 2015. Kaiser Family Foundation. Available at: http://kff.org/medicare/report/medicare-part-dat-ten-years-the-2015-marketplace-and-key-trends-2006-2015/. Accessed January 28, 2017.

22. Pearson CF. Few medicare beneficiaries receive comprehensive medication review services. Avelere Health. August 7, 2014. Available at: http://avalere. com/expertise/managed-care/insights/few-medicare-beneficiaries-receivecomprehensive-medication-management-serv. Accessed January 28, 2017.

23. Yap D. Under CMS proposed rule, MTM coverage would expand greatly. Pharmacy Today. January 14, 2014. Available at: https://www.pharmacist. com/node/425354?is_sso_called=1x. Accessed January 28, 2017.

24. Kreuter MW, Strecher VJ. Do tailored behavior change messages enhance the effectiveness of health risk appraisal? Results from a randomized trial. Health Educ Res. 1996;11(1):97-105.

25. Tse B, Augustine J, Boesen K. Impact of a nationwide medication therapy management program on drug-related problems at the Medication Management Center in 2012. J Am Pharm Assoc. 2014;54(2):el41 [Abstract].

26. Howdent LM, Meyer JA. Age and sex composition: 2010. 2010 Census Briefs. U.S. Census Bureau. May 2011. Available at: http://www.census.gov/ prod/cen2010/briefs/c2010br-03.pdf. Accessed January 28, 2017. 